\title{
FDA Device Problem Code Hierarchy
}

National Cancer Institute

\section{Source}

National Cancer Institute. FDA Device Problem Code Hierarchy. NCI Thesaurus. Code C54451.

Terminology used for defining mode of device failure or malfunction in individual case reports for voluntary and mandatory reporting of adverse reactions or adverse events during both pre and post approval periods of device development and usage. 